\title{
Aftereffect of high-speed motion
}

Frans A J Verstraten, Maarten J van der Smagt, Wim A van de Grind

Helmholtz Instituut and Comparative Physiology, Universiteit Utrecht, Padualaan 8,

NL 3584 CH Utrecht, The Netherlands; e-mail: f.a.j.verstraten@bio.uu.nl

Received 25 February 1998, in revised form 9 July 1998

\begin{abstract}
A visual illusion known as the motion aftereffect is considered to be the perceptual manifestation of motion sensors that are recovering from adaptation. This aftereffect can be obtained for a specific range of adaptation speeds with its magnitude generally peaking for speeds around $3 \mathrm{deg} \mathrm{s}^{-1}$. The classic motion aftereffect is usually measured with a static test pattern. Here, we measured the magnitude of the motion aftereffect for a large range of velocities covering also higher speeds, using both static and dynamic test patterns. The results suggest that at least two (sub)populations of motion-sensitive neurons underlie these motion aftereffects. One population shows itself under static test conditions and is dominant for low adaptation speeds, and the other is prevalent under dynamic test conditions after adaptation to high speeds. The dynamic motion aftereffect can be perceived for adaptation speeds up to three times as fast as the static motion aftereffect. We tested predictions that follow from the hypothesised division in neuronal substrates. We found that for exactly the same adaptation conditions (oppositely directed transparent motion with different speeds), the aftereffect direction differs by $180^{\circ}$ depending on the test pattern. The motion aftereffect is opposite to the pattern moving at low speed when the test pattern is static, and opposite to the high-speed pattern for a dynamic test pattern. The determining factor is the combination of adaptation speed and type of test pattern.
\end{abstract}

\section{Introduction}

After adaptation to a moving pattern for some time, a stationary pattern appears to move in the opposite direction for a while. This illusory motion is known as the motion aftereffect (MAE) or the Waterfall Illusion (Thompson 1880; Wade and Verstraten 1998). The MAE can be induced by adaptation to patterns moving with a relatively broad range of speeds. Several researchers have investigated the relation between the magnitude of MAE and adaptation speed. In most cases, the magnitude (eg duration) can be described as an inverted- $U$-shaped function of the adaptation speed. Thompson (1993) reviewed some of the studies on the effects of adaptation speed and reported that, in general, the optimal adaptation speed is about $2-3 \mathrm{deg} \mathrm{s}^{-1}$ (see also Scott and Noland 1965; or Ashida and Osaka 1995 for a different result).

However, a recent finding provides good reasons to believe that the idea of a single curve representing the magnitude of MAE as a function of adaptation speed is too simple. Verstraten et al (1999) had their subjects adapt to transparent motion; two patterns with different speeds were moving through each other in orthogonal directions (see also figure 4a). One pattern had a low speed and the other a higher speed (in their case $2 \mathrm{deg} \mathrm{s}^{-1}$ and $8 \mathrm{deg} \mathrm{s}^{-1}$ ). After adaptation, a test pattern was presented that was either static (a stationary random-dot pattern) or dynamic (a display in which pixels are randomly refreshed, which looks like a detuned TV). The observers' task was to indicate the direction of MAE. Normally, a MAE of transparent motion has only one direction (eg Mather 1980; Verstraten et al 1994). Surprisingly, for exactly the same adaptation configuration, the perceived direction of MAE differed on average by as much as $50^{\circ}$ with a change of the type of the test pattern. That is, the MAE stayed unidirectional but its direction was different depending on the type of test pattern. In general, when a dynamic test pattern was used, the direction of MAE was nearly opposite 
to that of the high-speed pattern, and when a static pattern was used, the direction of MAE was more opposite to that of the low-speed pattern.

If the speed that produces the optimal magnitude of MAE is the sole determining factor (largest magnitude is achieved at $2-3 \mathrm{deg} \mathrm{s}^{-1}$ as mentioned above ${ }^{(1)}$ ), the pattern of near-optimal speed would be expected to dominate the direction of MAE under all circumstances. Or, if all motion sensor signals were pooled during adaptation to obtain one common gain for the resultant vector direction and the test stimulus were to read out this common integrative stage, one would always expect the same direction of MAE regardless of the test stimulus. In other words, the direction of MAE should predominantly be determined by the more optimal adapting vector, for both test conditions. As mentioned earlier, this is not what has been found in our previous experiment. Under dynamic test conditions, adapted sensors coding for higher speeds dominate the direction of MAE. Obviously, there must be a MAE produced by sensors tuned to higher speeds that does not show itself under classic test conditions.

Until fairly recently, classic conditions for obtaining a MAE meant testing with static patterns. Several researchers have pointed out that dynamic test patterns show previously undiscovered characteristics of MAE (eg Green et al 1983; von Grünau 1986; Hiris and Blake 1992; Ledgeway 1994; Nishida and Sato 1995; Verstraten et al 1996a). On the basis of other psychophysical findings in the domain of motion perception (eg van de Grind et al 1986, 1994), we have recently suggested that low and high speeds are initially processed separately by different populations or in different channels (Verstraten et al 1999). More specifically, we have suggested that slow and fast units might contribute differently to the MAE, depending on the nature of the test pattern. This hypothesis is tested in experiment 1 . The idea is that if two different sensor groups (channels) underlie the aftereffect of transparent motion with their dominance depending on the type of test condition, they must have different 'tuning' curves.

We indeed found two different tuning curves for static and dynamic MAEs. A prediction that follows from that result is tested in experiment 2.

\section{Experiment 1}

In the first experiment we measured the magnitude of MAE for a range of adaptation velocities using two different types of test patterns-static and dynamic.

\subsection{Methods}

2.1.1 Stimulus generation. A random-pixel array (RPA) pattern (see figure 1) was generated by a custom-built hardware noise-pattern generator controlled by a Macintosh computer. The display contained $256 \times 256$ pixels and was $14 \mathrm{~cm} \times 14 \mathrm{~cm}$ square $(1$ pixel $\approx 0.55 \mathrm{~mm}$ ). Each pixel on the screen corresponded to one pixel on the CRT raster. The average luminance was set to $50 \mathrm{~cd} \mathrm{~m}^{-2}$ and the rms contrast was held at $70 \%$. Patterns were presented on a CRT display (ElectroHome model EVM-1200, P4 phosphor) at a display rate of $90 \mathrm{~Hz}$. A more detailed description has been given in previous papers (eg Fredericksen et al 1993).

2.1.2 Procedure. Observers adapted for $30 \mathrm{~s}$ to a moving RPA. The pattern was moving horizontally either from left to right or vice versa (see figure 1). The speeds ranged from $0.35 \mathrm{deg} \mathrm{s}^{-1}$ ( 1 pixel step every 8 frames) to $79 \mathrm{deg} \mathrm{s}^{-1}$ (steps of 28 pixels every frame). After adaptation, a test pattern was shown and the MAE duration was measured with the use of the computer keyboard and clock. Although the use of duration as a measure of the magnitude of MAE is controversial (eg Anstis 1987; Pantle 1998), for this experiment it is most appropriate. The method is fast and we are not interested in small differences for which more sensitive techniques may be required. The test 


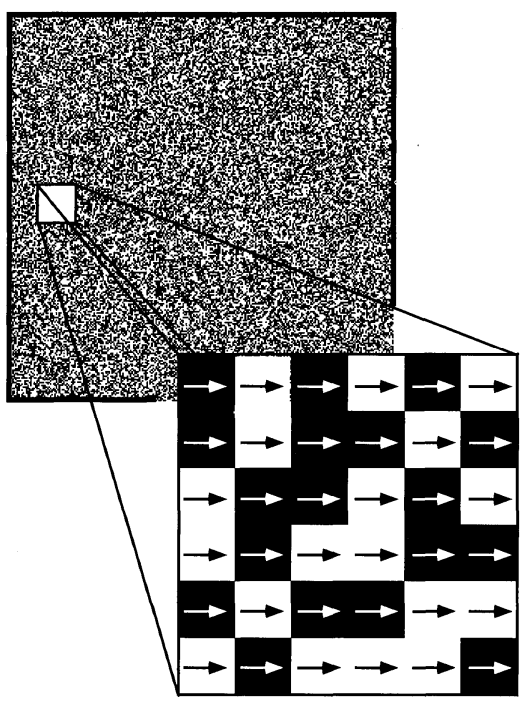

Figure 1. Example of a random-pixel array (RPA). The inset shows a sample of the pixels. In a RPA, all pixels make the same step size every $x$ frames. Although several terms have been used in the past, we prefer to use RPA because it is a special case of a randomdot pattern. In our stimulus the initial randomly drawn $256 \times 256$ pixels are moving as a coherent pattern, an 'array', not as individual dots, appearing and disappearing only at the sides of the display.

pattern could be either static visual noise (a stationary RPA) or dynamic visual noise. When dynamic noise was presented, its temporal-frequency cutoff was $45 \mathrm{~Hz}$ (which means that all the pixels were randomly refreshed every $22 \mathrm{~ms}$ ). After the subject indicated the duration or absence of MAE, a $30 \mathrm{~s}$ pause was given. The static and dynamic test conditions were presented in a pseudo-randomised order and so were the velocities. Viewing distance was $1 \mathrm{~m}$, resulting in a square display of area $8 \mathrm{deg} \times 8 \mathrm{deg}$, in a dimly lit room. Viewing was binocular and a fixation point was present in the centre of the display. Head support and a chin-rest were provided. All conditions were presented sixty-four times ( 2 directions $\times 2$ test patterns $\times 16$ velocities).

2.1.3 Observers. Two experienced observers (the authors MS and FV) participated in this experiment.

\subsection{Results and discussion}

The results for both observers are shown in figure 2, where the duration of MAE is plotted as a function of the adaptation speed. We collapsed the data for 'equal' speeds with opposite motion directions (see procedure). Several observations can be made. The data show that the dynamic MAE is perceived over a much broader range extending to higher speeds. For our stimulus, the static MAE is not present for speeds higher than approximately $20 \mathrm{deg} \mathrm{s}^{-1}$. The dynamic MAE is still visible for speeds about three times as fast. At the lower end of the speed range the magnitude of MAE is larger for a static test pattern than that for a dynamic test pattern. The opposite is true for the higher end of the speed range where the dynamic MAE is more prevalent.

One additional observation should be mentioned. In one condition a dynamic test pattern was presented after adaptation to a low-speed pattern, where low is defined as a speed to the left of the equality point $\mathrm{E}$ in figure $3 \mathrm{a}$ (equality point stands for the speed at which static and dynamic MAEs have the same magnitude). The duration of the dynamic MAE was subsequently measured. As soon as the observer indicated that the MAE had disappeared, the dynamic pattern was replaced by a static pattern. At that instant the MAE showed up again. This phenomenon has been reported previously (Verstraten et al 1996b). However, we now report that it only seemed to happen for the cases when dynamic testing preceded a static pattern after adaptation to lower speeds. Dynamic testing after adaptation to higher speeds followed by a static test pattern did not result in the reappearance of a MAE. We are currently investigating this in more detail and preliminary results have been reported by van der Smagt et al (1998). 


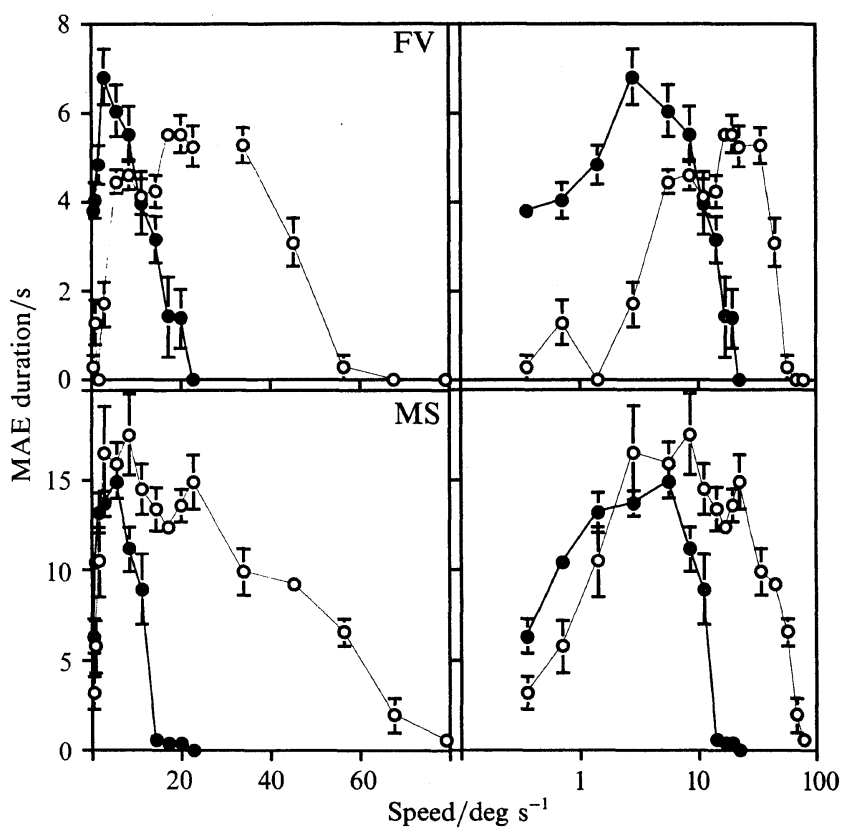

Figure 2. Results for two subjects. MAE duration is plotted as a function of the adaptation speed. The curve with the solid symbols represents MAE duration obtained with a static test pattern. The data obtained with a dynamic test pattern are represented by the open symbols. The abscissa (speed) has a linear scale in the left panels. In order to give a more detailed illustration of the lower end of the speed range it is logarithmic in the right panels.

For now, we tentatively conclude that for a given direction there are at least two pools of sensors underlying the different MAEs. One pool that is predominantly tuned to the lower end of the speed range and another pool that is more sensitive to higher speeds (strictly speaking, more effective in inducing static and dynamic MAEs, respectively). Given this, some predictions follow, which are tested in experiment 2.

\section{Experiment 2}

In figure $3 a$ we have schematically represented the findings of experiment 1 . To the left of the equality point $\mathrm{E}$ the static MAE is stronger than the dynamic MAE ('static MAE dominant'). To the right of $E$ the dynamic MAE is stronger than the static MAE. For a part of this 'dynamic MAE dominant' range, the static MAE does not exist. This range is called 'dynamic MAE only'. It is harder to localise an analogous range at the lower speeds for static MAEs ('static MAE only'). The upper part of the gray area in figure $3 a$ represents the minimal duration of MAE for what we consider a reliable magnitude of MAE. We chose this, arbitrary, threshold level at approximately one-third of the maximum value of MAE.

The curves obtained in experiment 1 show that MAEs for high adaptation speeds exist but can only be made visible by dynamic test patterns. As stated before, we hypothesise that different populations of sensors underlie these MAEs. This allows us to formulate and test a prediction for the MAE of transparent motion. By transparent motion we refer to the situation where two velocity vectors are present and are perceived separately in the same part of the visual field (eg Braddick 1997).

Let us construct transparent motion consisting of a low-speed and a high-speed component. We can choose the low speed such that it will produce a strong static MAE and a weak or no dynamic MAE. In the same vein we can choose a high speed that results in a strong dynamic MAE and no or a weak static MAE. After adaptation to transparent motion of both speeds, we expect a MAE opposite to the low-speed pattern for a static test pattern, and a MAE opposite to the high-speed pattern for a dynamic test pattern. That is, for oppositely directed transparent motion, we expect MAEs that differ $180^{\circ}$ in direction depending only on the type of the test pattern. 


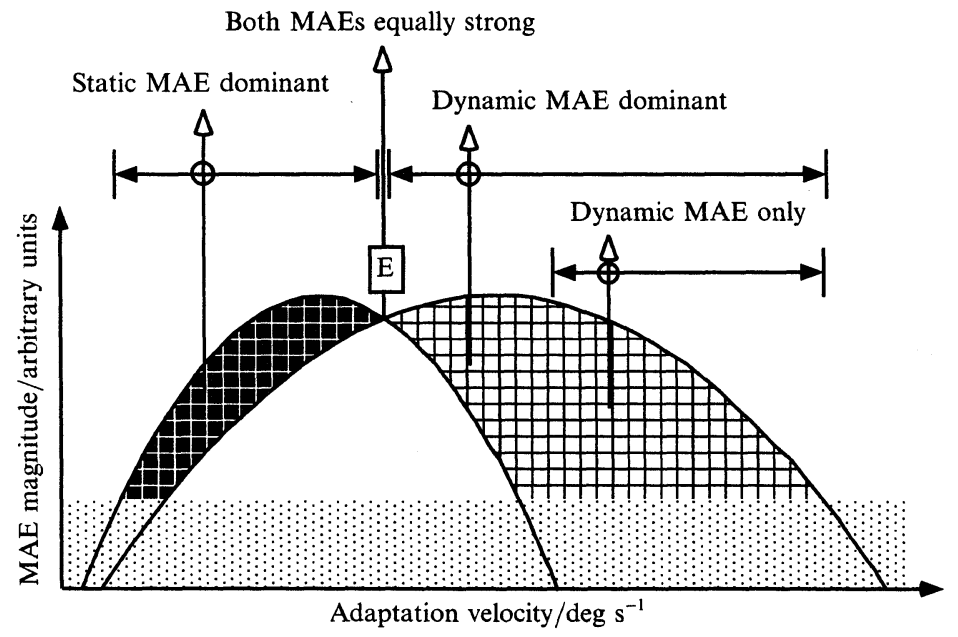

(a)
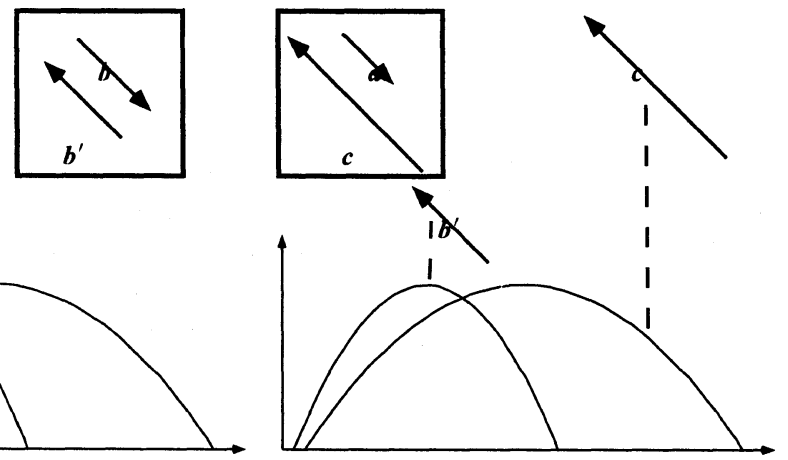

Velocity 2

(b)

Velocity 1

Figure 3. (a) Schematic representation of the curves as found in experiment 1 . Left of equality point $E$ the static MAE is stronger than the dynamic MAE (static MAE dominant). On the right side of $\mathrm{E}$ the dynamic MAE is stronger than the static MAE. For a subset of this 'dynamic MAE dominant' range, the static MAE does not exist. This range is called 'dynamic MAE only'. It is hard to localise an analogous range at lower speeds for static MAEs. (b) It is important to note that in case of transparent motion in opposite directions (or in general for different directions), two sets of two 'tuning' curves have to be considered to allow predictions about the direction of the resulting MAE. For example, in the case of transparent motion in opposite directions with equal speed as represented by vectors $\boldsymbol{b}$ and $\boldsymbol{b}^{\prime}$, the MAE will be a product of two populations tuned to different directions. One population tuned to direction $\boldsymbol{b}$ and the other to direction $\boldsymbol{b}^{\prime}$ but with the same speed-tuning characteristics for a given speed. For transparent motion of slow and fast speeds as represented by vectors $\boldsymbol{a}$ and $\boldsymbol{c}$, this idea becomes more important to understand the result.

For opposite adaptation directions the stronger (longer) MAE always determines the direction of MAE. To be able to see also small contributions by the non-dominant component (if present), we also present orthogonally directed transparent motion. The difference in perceived direction between static and dynamic MAEs for these adaptation conditions can be as large as $90^{\circ}$.

\subsection{Methods}

3.1.1 Stimulus generation. Apparatus and setup were the same as for experiment 1. Transparent motion was established by spatial transparency (eg van Doorn and Koenderink 1982). More specifically, we used spatial 'checkerboard' transparency: a checkerboard pattern of contiguous windows $(1 \times 1$ pixels on CRT raster) displaying the two patterns (see Verstraten et al 1994 and figure 4). 

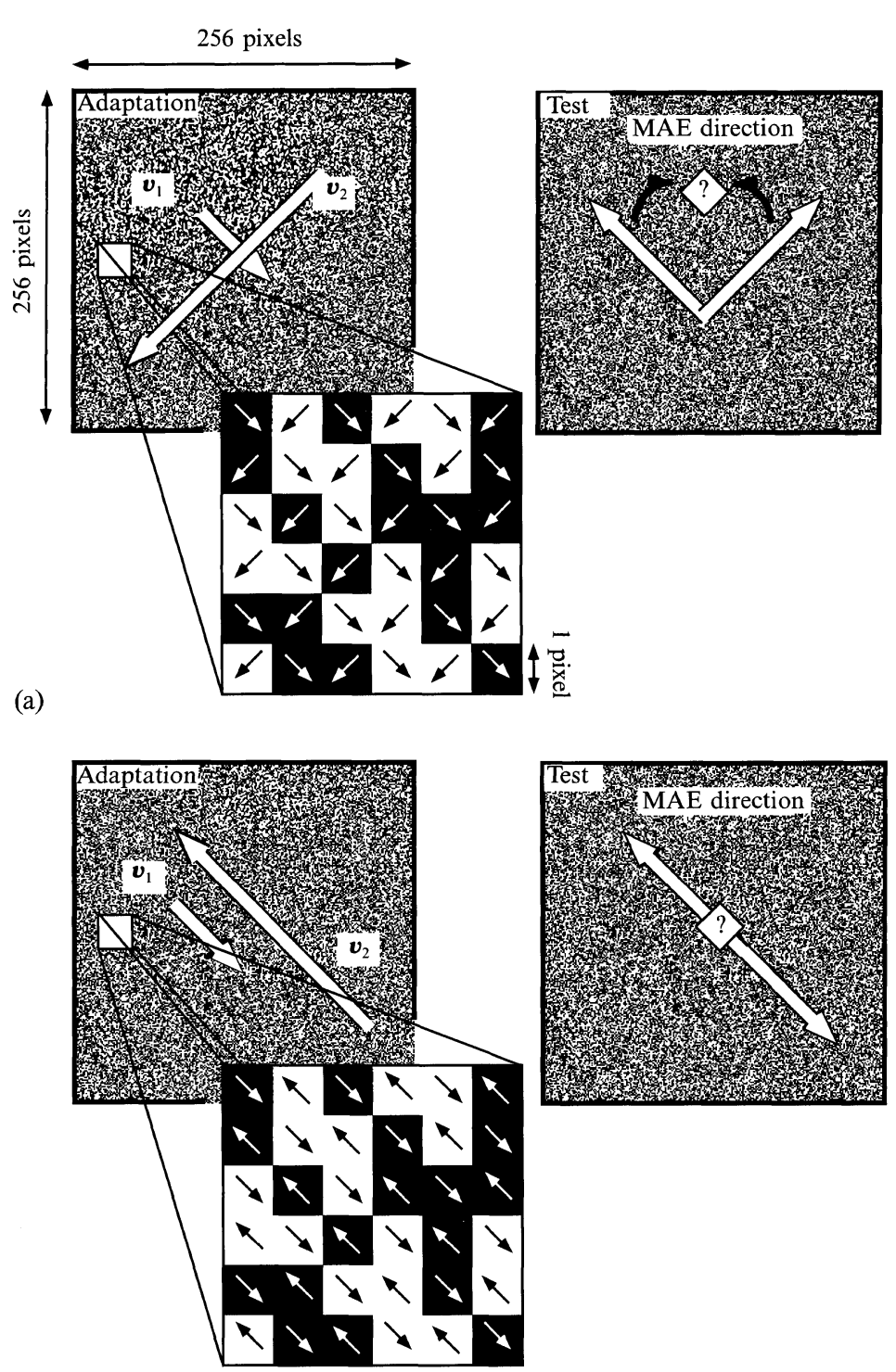

(b)

Figure 4. Transparent motion for (a) orthogonal directions and (b) opposite directions. The insets show the way we generate transparent motion: a checkerboard pattern of contiguous windows $(1 \times 1$ pixels $)$.

3.1.2 Procedure. In this experiment, there were two main conditions. In the first condition a low and a high speed opposite in direction were presented under transparentmotion conditions. It is important to understand that during adaptation this stimulus will activate sensors tuned to different directions. Therefore, different tests will tap sensors tuned not only to different speeds but also to different directions (see figure $3 \mathrm{~b}$ vectors $\boldsymbol{a}$ and $\boldsymbol{c}$ ). In the second condition, orthogonally directed transparent motion was used with the same speeds (see below for details).

A simple control condition was added as well. In the case of opposite transparent motion, when both adapting vectors have the same speed and therefore lead to the same magnitude of MAE, a cancellation of the MAE is expected for both static and dynamic test patterns as long as the speed is represented in both the static and the dynamic 
'tuning' curve. For orthogonal transparent motion with equal speeds the MAE should be exactly opposite to the vector sum of the two inducing patterns, again irrespective of the type of the test pattern.

Transparent motion in orthogonal directions. Observers adapted for $30 \mathrm{~s}$ to two simultaneously presented RPAs moving transparently in orthogonal directions. The patterns were moving in the $225^{\circ}$ and $315^{\circ}$ directions (anticlockwise from $0^{\circ}$, which is the 3 o'clock direction, see figure 4 and figure 5). The speeds of the two inducing patterns were chosen for each subject individually, on the basis of results of experiment 1. For subject MS we chose $0.66 \mathrm{deg} \mathrm{s}^{-1}$ (vector $\boldsymbol{a}$, see figure $3 \mathrm{~b}$ ) as the low speed and $32 \mathrm{deg} \mathrm{s} \mathrm{s}^{-1}$ (vector $\boldsymbol{c}$ ) as the high speed. We chose $4 \mathrm{deg} \mathrm{s}^{-1}$ (vectors $\boldsymbol{b}$ and $\boldsymbol{b}^{\prime}$ ) for the equal-speed condition (for this speed both static as well as dynamic tests give good MAEs). For subject FV these values were $2 \mathrm{deg} \mathrm{s}^{-1}, 32 \mathrm{deg} \mathrm{s}^{-1}$, and also $4 \mathrm{deg} \mathrm{s}^{-1}$, respectively (these values are given in the table in the lower part of figure 5).

After $30 \mathrm{~s}$ of adaptation a test pattern was shown for $2 \mathrm{~s}$. This test pattern could either be static visual noise (SVN, a stationary RPA) or dynamic visual noise (DVN, see section 2.1). After the test pattern was shown, a horizontal black line, 1 pixel wide was presented on an RPA that was always static. One could argue that it would be better to present the line on a nontextured average-luminance display. In a previous experiment we tested this for several observers and there were no significant differences

$\mathrm{A}_{1}$
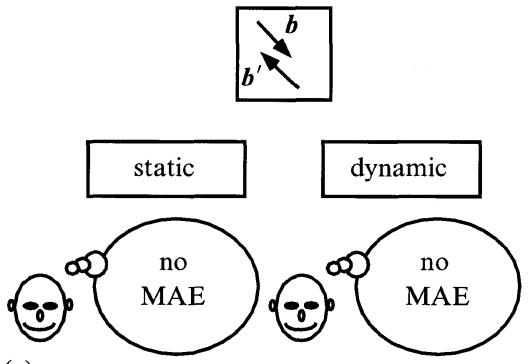

(a)

$\mathrm{B}_{1}$
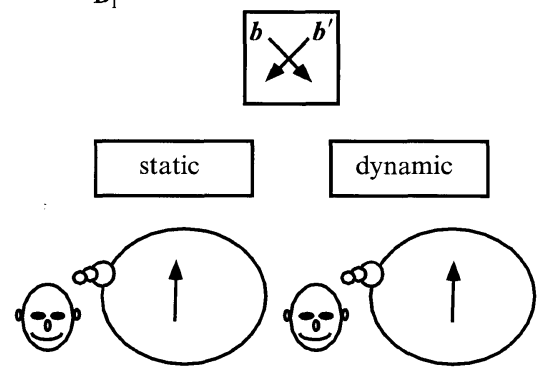

Subject

$\frac{\text { Speed } / \mathrm{deg} \mathrm{s}^{-1}}{a \quad b \quad c} \quad \begin{aligned} & \text { Direction of } \\ & \text { static MAE }^{\circ}\end{aligned}$

MS

(b)

$\begin{array}{lll}0.66 & 4 & 32\end{array}$

134.13

131.25

Test

Percept

Adaptation stimulus

3.09

2.63

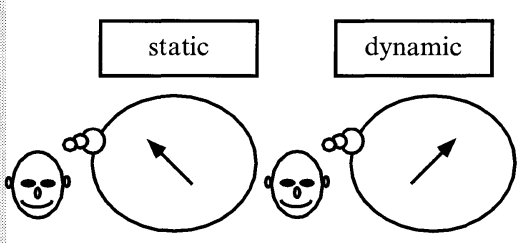

$\mathrm{B}_{2}$
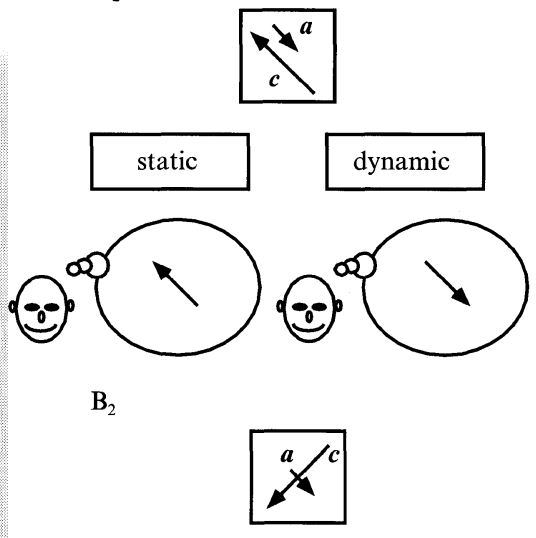

$\mathrm{A}_{2}$

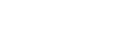

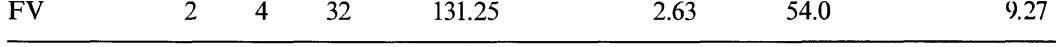

Figure 5. (a) Illustration of the results for adaptation to oppositely directed transparent motion for both static and dynamic test patterns and vectors with $\left(\mathrm{A}_{1}\right)$ equal speed $\left(4 \mathrm{deg} \mathrm{s}{ }^{-1}\right.$, see table in the lower part of the figure), and $\left(\mathrm{A}_{2}\right)$ with a slow and a fast speed. (b) Results for transparent motion in orthogonal directions: $\left(B_{1}\right)$ for the same speeds, and $\left(B_{2}\right)$ for a combination of low and high speeds. The table shows the average direction of the MAE for the $\mathrm{B}_{2}$ condition. 
(Verstraten et al 1999). Observers were asked to adjust the orientation of the line such that it was parallel to the direction of the MAE as they experienced it, immediately after the test pattern was presented. After the observer had indicated the perceived direction, the final value was stored by the computer, and followed by a $30 \mathrm{~s}$ pause. The static and the dynamic test conditions were presented in a random order. Viewing conditions were the same as in experiment 1.

Transparent motion in opposite directions. The patterns were moving along the diagonal with the same set of speeds as for the orthogonal-direction condition (see figure 5a). After adaptation, either a static or dynamic test pattern was presented and observers had to indicate only whether the MAE was moving to the upper left, lower right, or not at all. All conditions were repeated four times.

\subsection{Results}

The results for both subjects are illustrated in figure 5. We collapsed the data for equivalent adaptation combinations. For three out of four conditions it is sufficient to describe and illustrate the results in a qualitative manner. In the upper part of figure 5, adaptation to oppositely directed transparent motion is represented. If both patterns have the same speeds there is no MAE for either test pattern (condition $A_{1}$ ). When the vectors have different speeds (condition $A_{2}$ ), the MAE behaves as expected from the curves reported in experiment 1 . When the test is static, the MAE is opposite to the slow pattern; and when the test pattern is dynamic, the MAE is opposite to the fast pattern-a difference of $180^{\circ}$.

In the lower panel of figure 5 the orthogonal adaptation conditions are shown. As expected, the MAE of vectors with the same speed is opposite to the vector sum of the adapting directions (condition $\mathrm{B}_{1}$ ). Where the vectors have different speeds, the directions are basically opposite to the dominating inducing directions, as expected from our two (sub)population hypotheses. The speeds used in all the conditions and the quantitative results (the direction of MAE) for the $B_{2}$ condition are presented in the table in the lower part of figure 5.

\section{General discussion}

In this paper we have shown that the range of speeds that generate MAEs is larger than expected on the basis of the classic MAE (defined as the MAE tested with a stationary test pattern). If a dynamic test pattern is used, the illusory motion can be seen for adaptation speeds up to three times faster than the upper velocity limit for generating a perceivable static MAE. We postulated a division into different pools of detectors within the population of motion sensors to account for this finding. A simple test in experiment 2 provided support for this idea: when an observer adapts to oppositely directed transparent motion, consisting of a low-speed and high-speed pattern, for the same adaptation conditions the direction of MAE differs by $180^{\circ}$, depending on the type of the test pattern. If a static test is used, the MAE is opposite to the slow adaptation pattern and if the test pattern is dynamic the MAE is opposite to the fast pattern. The same rule holds for adaptation to orthogonal transparent motion.

In this discussion we would like to elaborate on the following three topics:

(1) the concept of 'two populations' of motion sensors,

(2) how this work relates to recent research on MAEs, and

(3) what the possible implications are for models of MAE.

\subsection{Separate populations of sensors tuned to high-velocity and low-velocity motion?}

There are several independent indications that separate parallel streams process slow and fast motions. A complete survey of the relevant literature would be out of place here. Below, we discuss some of the evidence and assume that these selective references suffice to establish that the two-channel idea is not too wild a hypothesis. 
One report that might be related involves a famous cerebral akinetopsia patient (see Zihl et al 1983). This person has bilateral brain damage that includes the putative human analogue for the middle-temporal area (MT/V5) and is known to have severe problems in perceiving motion. These problems appear selective and seem to be restricted to the higher speed range. Hess et al (1989) showed that she was unable to perform a speed-discrimination task when the speeds were above $6 \mathrm{deg} \mathrm{s}^{-1}$. For this patient the human analogue for V5 is not available for visual motion processing, so there must be other neural substrates for processing slow motion. On the basis of a PET (positron emission tomography) study by Shipp et al (1994), Gegenfurtner and Hawken (1996) suggested a possible pathway through areas V3 and V4 for slow motion.

Gegenfurtner and Hawken (1995, see also 1996), using different stimuli from ours, also suggest two processing streams for motion. One for fast motion and one for slow motion, where the difference is based on temporal characteristics (although in their case low speeds were not veridically perceived). Differences between channels based on temporal characteristics have received considerable attention in the past. Kulikowski and Tolhurst (1973) were among the first to explicitly differentiate between channels based on temporal characteristics. They related sustained versus transient cells to pattern versus motion channels. However, several subsequent papers suggested that both transient as well as sustained channels carry motion information (eg Thompson 1983; Anderson and Burr 1985), again showing that two channels are likely to be involved in motion processing.

It is also known that motion sensors that are tuned to different speeds have different characteristics. For example, van de Grind et al (1986) showed that bilocal-type motion detectors coding for low speeds are tuned by their delay, those coding for higher speeds by their span (see also van de Grind et al 1994). Mutual-suppression and related studies supply more and more evidence for two motion channels, one for low and one for high speeds (eg Snowden 1990; Verstraten et al 1996b; Edwards et al 1998).

Although the classic notion is that parvocellular (P) neurons deal with colour and form information and magnocellular (M) neurons with motion processing, Livingstone and Hubel (1987) also suggested that the sustained channel ( $P$ pathway) mediates slowly moving patterns. This indicates that both $\mathbf{P}$ and $\mathbf{M}$ pathways are involved in motion processing. Merigan and Maunsel (1993) hold a more recent view: Merigan (1998, personal communication) suggests that both pathways are involved as long as the motion falls in the spatiotemporal range of the $\mathrm{P}$ and $\mathrm{M}$ pathways.

So there are several reports indicating differences between processing of high and low speeds. In a previous paper we used the term sustained (S) channel for lowvelocity-tuned motion sensors and transient $(T)$ channel for high-velocity-tuned motion sensors (Verstraten et al 1999). Although we used this for ease of exposition, this distinction is more common in recent motion literature (eg Strout et al 1994; Wolf and Lusty 1994). In any case, the present findings provide support for the idea of at least two, possibly parallel, channels or even pathways, one consisting of motion sensors mainly tuned to higher speeds and the other of units mainly tuned to lower speeds.

\subsection{Relation to recent research on MAEs}

As we have briefly discussed in the introduction, the difference between static and dynamic MAEs has become of much interest recently. Vision scientists have found differences depending on the kind of test patterns in the past (eg Green et al 1983), but the first experimental and theoretical emphasis came from von Grünau (1986) and Cavanagh and Mather (1989), respectively. Recent reports have provided evidence suggesting that static and dynamic MAEs arise at different levels of visual motion processing (eg Culham and Cavanagh 1994; Nishida et al 1994; Nishida and Sato 1995; Ashida and Verstraten 1998; see also Raymond 1993). Often special kinds of motion were used, like first-order and second-order motion (Chubb and Sperling 1988; Cavanagh 
and Mather 1989). We, on the other hand, have focused on static and dynamic MAEs of luminance-contrast-based motion, which is predominantly first order (as did Hiris and Blake 1992; see also von Grünau and Dubé 1992). Of course, there is always secondorder information in the stimulus but there is no reason to believe that this information can account for the present results.

So far it has been assumed that if adaptation to first-order motion results in a static MAE it will also show a dynamic MAE. We have shown that this is not necessarily so. For higher speeds, MAEs of first-order motion can only be perceived if the test pattern is dynamic. We note that, while investigating another phenomenon, Wainwright and Cavanagh (1999) also were able to generate a dynamic MAE while there was no static MAE for adaptation to luminance-contrast-based motion.

In a way our results seem to conflict with those of Grunewald and Lankheet (1996). Basing themselves on a model by Grunewald (1996), they presented psychophysical evidence that oppositely directed transparent motion results in an orthogonal MAE. More recently they reported that this MAE is actually bidirectional (Grunewald and Lankheet 1997). These aftereffects are not perceived with our stimuli, possibly because our RPAs are high-density rather than sparse dot stimuli. This discrepancy needs further investigation.

\subsection{Implications for MAE models}

Although there were some ideas about the mechanisms underlying MAE in the 19th century (see Wade and Verstraten 1998), the first explicit model was presented in the early sixties of this century and became known as the ratio model (Sutherland 1961; Barlow and Hill 1963). The strict ratio model was followed by Mather's distributionshift model (Mather 1980; see also Levinson and Sekuler 1976). These models have their problems, which are discussed in Mather and Harris (1998).

As an alternative, Mather and Harris use the model of Wilson and his colleagues (eg Wilson et al 1992; Wilson and Kim 1994). This model seems promising since it can handle motion in two dimensions (eg Exner 1887; Adelson and Movshon 1982) as well as some higher-order MAE phenomena. One of the reasons that Wilson's model is especially interesting for MAE research is because it potentially allows adaptation to occur at multiple sites. And this is what is required to explain the current results.

Wilson's model distinguishes between three layers. Starting from the front end these are: a sensor layer, an opponent-energy layer, and a layer of integrator units. As mentioned above, the model could potentially allow adaptation at all levels. For a transparentmotion stimulus, the directions are processed separately during adaptation. The fact that we can get an aftereffect direction depending on the type of test pattern can only be explained if the activity/sensitivity distributions along the pathway of visual motion processing are still separately available at the time the test stimulus is presented. The site of adaptation must be located either at the sensor level or at the opponent-stage level, but not at the final integrative level. Wilson considers motion transparency (transparent motion) as a "relatively infrequent phenomenon" (1994, page 246), but we would argue that transparent motion is a rather frequent percept. For example, when we see poles, fences, bushes, and the like at different distances while running, or shadows that move across moving objects, or while driving a car when rain pours on the front window, etc (see also Braddick 1997). Wilson's model would undoubtedly gain wider acceptance if it was able to handle transparent motion and its $\mathrm{MAE}(\mathrm{s})$.

Acknowledgements. We thank Hiroshi Ashida, Takao Sato, and Satoshi Shioiri for helpful discussion. FV is supported by a fellowship of the Royal Netherlands Academy of Arts and Sciences and MS by the Netherlands Organization for Scientific Research. Parts of this paper were written while FV was visiting the ATR Human Information Processing Research Laboratories in Kyoto, Japan. Their hospitality is gratefully acknowledged. 


\section{References}

Adelson E H, Movshon J A, 1982 "Phenomenal coherence of moving visual patterns" Nature (London) $300523-525$

Anderson S J, Burr D, 1985 "Spatial and temporal selectivity of the human motion detection system" Vision Research $251147-1154$

Anstis S, 1986 "Motion perception in the frontal plane: sensory aspects", in Handbook of Perception and Human Performance Eds J P Thomas, K R Boff, L Kaufman (New York: John Wiley)

Ashida H, Osaka N, 1995 "Motion aftereffect with flickering test stimuli depends on adapting velocity" Vision Research 351825 - 1834

Ashida H, Verstraten F A J, 1998 "Attentive tracking of motion: interaction between low-level and high-level motion systems", in Selection and Integration of Visual Information: Proceedings of the International Workshop on Advances in Research on Visual Cognition (Tsukuba, Japan: Science and Technology Association) pp 229-233

Barlow H B, Hill R M, 1963 "Evidence for a physiological explanation of the waterfall phenomenon and figural after-effects" Nature (London) $2001345-1347$

Braddick O, 1997 "Local and global representation of velocity: transparency, opponency, and global direction perception" Perception $26995-1010$

Cavanagh P, Mather G, 1989 "Motion: The long and short of it" Spatial Vision $4103-129$

Chubb C, Sperling G, 1988 "Drift-balanced random stimuli: A general basis for studying nonFourier motion perception" Journal of the Optical Society of America A 5 1986-2007

Culham J, Cavanagh P, 1994 "Attentive tracking of a counterphase grating produces a motion aftereffect" Investigative Ophthalmology \& Visual Science 35(4) 1622

Doorn A J van, Koenderink J J, 1982 "Spatial properties of the visual detectability of moving spatial white noise" Experimental Brain Research 45189 - 195

Edwards M, Badcock D R, Smith A T, 1998 "Independent speed-tuned global-motion systems" Vision Research 38 1573- 1580

Exner S, 1887 "Einige Beobachtungen über Bewegungsnachbilde" Centralblatt für Physiologie 1 135- 140

Fredericksen R E, Verstraten F A J, Grind W A van de, 1993 "Spatio-temporal characteristics of human motion perception" Vision Research 331193 - 1205

Gegenfurtner K R, Hawken M J, 1995 "Temporal and chromatic properties of motion mechanisms" Vision Research $351547-1563$

Gegenfurtner K R, Hawken M J, 1996 "Pathways of motion and color in the visual pathways" Trends in Neuroscience $19394-401$

Green M, Chilcoat M, Stromeyer C F, 1983 "Rapid motion aftereffect seen within uniform flickering test fields" Nature (London) $30461-62$

Grind W A van de, Koenderink J J, Doorn A J van, 1986 "The distribution of human motion detector properties in the monocular visual field" Vision Research 26 797-810

Grind W A van de, Verstraten F A J, Zwamborn K M, 1994 "Ensemble models of the movement aftereffect and the influence of eccentricity" Perception $231171-1180$

Grünau M W von, 1986 "A motion aftereffect for long-range stroboscopic apparent motion" Perception \& Psychophysics 4031 - 38

Grünau M von, Dubé S, 1992 "Comparing local and remote motion aftereffects" Spatial Vision $6303-314$

Grunewald A, 1996 "A model of transparent motion and non-transparent motion aftereffects", in Neural Information Processing Systems 8 Eds D S Touretzky, M C Mozer, M E Hasselmo (Cambridge, MA: MIT Press) pp 837-843

Grunewald A, Lankheet M J M, 1996 "Orthogonal motion after-effect illusion predicted by a model of cortical motion processing" Nature (London) 384 358-360

Grunewald A, Lankheet M J M, 1997 "Bidirectional orthogonal motion aftereffect" Investigative Ophthalmology \& Visual Science 38(4) S216

Hess R F, Baker C L, Zihl H, 1989 “The 'motion blind' patient: low-level spatial and temporal filters" Journal of Neuroscience 9 1628- 1640

Hiris E, Blake R, 1992 "Another perspective on the visual motion aftereffect" Proceedings of the National Academy of Sciences of the USA $899025-9028$

Kulikowski J J, Tolhurst D J, 1973 "Psychophysical evidence for sustained and transient detectors in human vision" Journal of Physiology (London) 232 149-162

Ledgeway T, 1994 "Adaptation to second-order motion results in a motion aftereffect for directionally ambiguous test stimuli" Vision Research $342879-2889$

Levinson E, Sekuler R, 1976 "Adaptation alters perceived direction of motion" Vision Research $16779-781$ 
Livingstone M S, Hubel D H, 1987 "Psychophysical evidence for separate channels for the perception of form, color, movement and depth" Journal of Neuroscience 7 3416-3468

Mather G, 1980 "The movement aftereffect and a distribution-shift model for coding the direction of visual movement" Perception 9 379-392

Mather G, Harris J P, 1998 "Theoretical models of the motion aftereffect", in The Motion Aftereffect: A Modern Perspective Eds G Mather, F A J Verstraten, S Anstis (Cambridge, MA: MIT Press) pp $157-185$

Merigan W H, Maunsel J H R, 1993 "How parallel are the primate visual pathways?" Annual Review of Neuroscience 16 369-402

Nishida S, Ashida H, Sato T, 1994 "Complete interocular transfer of motion aftereffect with flickering test" Vision Research 34 2707-2716

Nishida S, Sato T, 1995 "Motion aftereffect with flickering test patterns reveals higher stage of motion processing" Vision Research 35 477-490

Pantle A, 1998 "How do motion aftereffect measures measure up", in The Motion Aftereffect: A Modern Perspective Eds G Mather, F A J Verstraten, S Anstis (Cambridge, MA: MIT Press) pp 25-39

Raymond J E, 1993 "Complete interocular transfer of motion adaptation effects on motion coherence thresholds" Vision Research 33 1865-1870

Scott T R, Noland J H, 1965 "Some stimulus dimensions of rotating spirals" Psychological Review $72344-357$

Shipp S, Jong B M de, Zihl J, Frackowiak R S, Zeki S, 1994 "The brain activity related to residual motion vision in a patient with bilateral lesions of V5" Brain 117 1023-1038

Smagt M J van der, Verstraten F A J, Grind W A van de, 1998 "Are motion aftereffects stored after adaptation to high speed motion?" Investigative Ophthalmology \& Visual Science 39(4) S1099

Snowden R J, 1990 "Suppressive interactions between moving patterns: Role of velocity" Perception \& Psychophysics 47 74-78

Strout J J, Pantle A, Mills S L, 1994 "An energy model of interframe interval effects in single-step apparent motion" Vision Research 34 3223-3240

Sutherland N S, 1961 "Figural aftereffects and apparent size" Quarterly Journal of Experimental Psychology $13222-228$

Thompson P, 1983 "Discrimination of moving gratings at and above detection thresholds" Vision Research $231533-1538$

Thompson P, 1993 "Motion psychophysics", in Visual Motion and Its Role in the Stabilization of Gaze Eds F A Miles, J Wallman (Amsterdam: Elsevier) pp 29-53

Thompson S P, 1880 "Optical illusions of motion" Brain 3 289-298

Verstraten FA J, Fredericksen R E, Grind W A van de, 1994 "The movement aftereffect of bivectorial transparent motion" Vision Research 34 349-358

Verstraten F A J, Fredericksen R E, Wezel R J A van, Boulton J C, Grind W A van de, 1996b "Directional motion sensitivity under transparent motion conditions" Vision Research 36 2333-2336

Verstraten F A J, Fredericksen R E, Wezel R J A van, Lankheet M J M, Grind W A van de, 1996a "Recovery from adaptation for dynamic and static motion aftereffects: Evidence for two mechanisms" Vision Research $36421-424$

Verstraten F A J, Smagt M J van der, Fredericksen R E, Grind W A van de, 1999 "Integration after adaptation to transparent motion: static and dynamic test patterns result in different aftereffect directions" Vision Research 39 (in press)

Wade N J, Verstraten F A J, 1998 "Introduction and historical overview", in The Motion Aftereffect: A Modern Perspective Eds G Mather, F A J Verstraten, S Anstis (Cambridge, MA: MIT Press) pp 1-23

Wainwright M, Cavanagh P, 1999 "Static and flicker motion aftereffects in a velocity space" (submitted)

Wilson H R, 1994 "Models of two-dimensional motion perception", in Visual Detection of Motion Eds A T Smith, R J Snowden (London: Academic Press) pp 219-251

Wilson H R, Ferrera V P, Yo C, 1992 "A psychophysically motivated model for two-dimensional motion perception" Visual Neuroscience 9 79-97

Wilson H R, Kim J, 1994 "A model for motion coherence and transparency" Visual Neuroscience $111205-1220$

Wolf J E, Lusty N G, 1994 "Rotating stripes provide a simultaneous display of sustained and transient channels" Spatial Vision 8 369-379

Zihl J, Von Cramon D, Mai N, 1983 "Selective disturbance of movement vision after the bilateral brain damage" Brain $106313-340$ 